\title{
Clinical profile of acutely ill psychiatric patients admitted to a general hospital psychiatric unit
}

\author{
ABR Janse van Rensburg \\ Division of Psychiatry, University of the Witwatersrand, Johannesburg, South Africa
}

\begin{abstract}
Objectives: Helen Joseph Hospital in southern Gauteng Province is one of five specialist hospitals on the academic circuit of the University of the Witwatersrand. Against a background of new mental health legislation, implemented in South Africa during December 2004 with no formal mechanisms in place to monitor mental health services on different levels of care or in regions, a study with three objectives was undertaken, namely: (I) to provide a baseline on psychiatric morbidity and treatment outcome; (II) to establish the state of affairs analysis for mental health care and (III) to establish a framework for cost centre management. The current study focuses on objective one. Method: A retrospective clinical audit was undertaken of mental health service delivery, teaching and research at Helen Joseph Hospital over a one-year period from September 2003 to August 2004 . This article reports on the two service delivery datasets identified: the "Inpatient Discharge Summary Report" and the "Consultation/Liaison Report". Results: A total number of 438 service users were admitted and a monthly average of 80 consultation/liaison assessments was conducted during the study period. Persistent unfavourable nursing staff ratios continued, while the number of service users from other African countries was generally underrated. Non-compliance and substance abuse contributed significantly to the admission of service users. Schizophrenia was indicated as the most likely diagnosis in almost a quarter of cases. Conclusion: Morbidity and treatment outcome at Helen Joseph Hospital will only be contextualized after the implementation of a regular clinical audit process in all the facilities of its referral network.
\end{abstract}

Key words: Psychiatric morbidity; Treatment outcome; Referral network; Clinical profile; Service delivery; Clinical audit

Received: $30-01-2006$

Accepted: 05-07-2006

\section{Introduction}

At the time of the study, mental health care in South Africa and Gauteng Province found itself in a critical period awaiting the imminent implementation of new legislation, the Mental Health Care Act, Act no 17 of 2002. Not only was change anticipated in the structures and procedures of mental health care delivery, but also in the whole human, physical and financial environment in which care is to be provided. While the typical bio-psycho-social model of mental health care provision was central to the existing referral system on primary, secondary and tertiary levels of care, the new legislation heralded an era

\section{Correspondence:}

Dr ABR Janse van Rensburg,

PO Box 1247 PINEGOWRIE Johannesburg 2123 South Africa

email: bernardj@gpg.gov.za of renewed emphasis on human rights in mental health care. The Act was eventually promulgated during December 2004. Mechanisms to monitor and assess the implementation of the new dispensation were however not yet in place at the time of the study. There was however a policy document by the quality assurance directorate of the provincial department of health, on general clinical audit principles and process for implementation in Gauteng hospitals. A structure allowing for a quality assurance committee at provincial level, as well as for clinical audit departments or committees at hospital level was suggested. A proposal pertaining to mental health services was submitted to the provincial department by the Division of Psychiatry of the University of the Witwatersrand. In this proposal it was agreed that psychiatric hospitals on the academic circuit such as the Tara and Sterkfontein hospitals should appoint their own hospital committees for this purpose. Services provided by acute psychiatric units in 
general hospitals on the circuit such as the Helen Joseph, Chris Hani Baragwanath and Johannesburg hospitals, should be covered by their particular hospital's committee. It was also suggested that the overall monitoring of the audit process in the different provincial sub-regions or across the different levels of service should be done by the different departments of Psychiatry of the three universities involved in the province - the University of the Witwatersrand, University of Pretoria and the Medical University of Southern Africa. With the confirmation of these structures and functions, it was foreseen that appropriate operational strategies would have to follow allowing for particular referral routes, a comprehensive clinical database, and appropriate time frames.

As a specific case scenario in question, Helen Joseph Hospital in southern Gauteng Province is a 457-bed facility and one of three general hospitals with an acute psychiatric unit on the service and teaching circuit of the Division of Psychiatry of the University of the Witwatersrand. The hospital is situated in southern Johannesburg but serves communities across the city including the eastern and western suburbs. Gauteng is one of nine provinces of South Africa with an estimated population of 8.8 million people according to the 2001 census, extended to an estimated 9.4 million due to cross-border flow from neighbouring provinces and countries. As a specialist level hospital both clinical and academic service delivery i.e. teaching of under and post graduate students and research formed part of the care scenario at Helen Joseph Hospital. Clinical service delivery at the time of the study consisted of a 30-bed inpatient unit for the psychiatric assessment and acute treatment of adults, an outpatient clinic and interdepartmental liaison and emergency consultation service. The unit accepted referrals from surrounding primary care clinics or hospitals and from ambulatory community psychiatric clinics. After screening and initial treatment, service users were referred to other specialized units such as the forensic, adolescent, psychotherapy and eating disorder units of the two psychiatric hospitals on the circuit.

No formal clinical audit department or committee existed at hospital level at the time of the study. Within Ward 2, the psychiatric unit, some quality improvement measures and access to clinical data about mental health care provided at the hospital were reviewed during September 2003. Systems and structures to monitor and report on care activities were adjusted and minimum data sets required for a supportive clinical database were incorporated in the discharge summary for inpatients and the consultation report form for interdepartmental consultations. Initial discussions with clinical staff were introduced on the possible benefits of appropriate reports on care conditions and outcome. Very limited support from the local information technology office was possible due to various reasons, with also no administrative support. As a quality in practice investigation, this study focused on mental health care at Helen Joseph as one of three psychiatric in-patient units at a general hospital in a network of referral levels and facilities on the circuit of the Division of Psychiatry in southern Gauteng.

This study had the following objectives: (i) to provide a baseline on psychiatric morbidity and treatment outcome in the population assigned to Helen Joseph Hospital for mental health care; (ii) to obtain a state of affairs analysis of mental health care and (iii) to provide a framework for future cost center management of mental health care. While the outcome of teaching, research and resource allocation is reported on separately, this article reports on the first objective stated, namely on morbidity and treatment outcome of the inpatient program and of the consultation/liaison services within Helen Joseph Hospital's referral system context. Ethical approval of the study was obtained from the Human Research Ethics Committee of the Faculty of Health Sciences, University of the Witwatersrand in August 2004.

\section{Method}

A retrospective clinical audit was undertaken of mental health service delivery, teaching and research functions performed at Helen Joseph Hospital over a period of one year from September 2003 to August 2004. Steps in the analysis of mental health care at this facility entailed the following: (i) Modeling of the functional areas according to service delivery, teaching and research and analyzing the activities within each area; (ii) Calculating the cost of each activity by establishing resources necessary to implement the program, while applying norms and standards for each functional area; and (iii) Analyzing data sets which accounts for both quantitative and qualitative outcomes of the different functional areas in the program. Service ("S"), teaching ("T") and research ("R") data sets were identified, including two service data sets for quantitative and qualitative outcomes: S1 - Inpatient Discharge Summary Report; and S2 Consultation/Liaison Report.

\section{Results}

\section{Service areas and activities}

INPATIENT CARE - Ward 2 is a 30-bed acute psychiatric admission and assessment unit for adults within a general hospital. A total of 438 service users were admitted during the study period, a monthly average of 36,5 . The average bed occupancy rate according to routine hospital statistics was $83 \%$ and the average length of stay was 18.5 days. The inpatient care program consisted of medical, psychological, psychiatric, functional and social examination and assessment of referred service users; initial containment and treatment; collection of collateral information; family intervention; education about psychiatric conditions and treatment; referral or placement after assessment; and reporting on treatment and recommendations.

CONSULTATION/LIAISON - A total of 799 consultation/liaison assessments were undertaken during office hours over 10 months of the study period. The average monthly total was 80 , with an average of 39 (49\%) per month from the casualty unit as emergency referrals. One doctor and one psychology intern were allocated to do routine and emergency consultations on a rotational basis. Psychiatric consultations included emergency assessments and management in the casualty department or routine consultations referred from medical, surgical and other departments. Routine consultations included assessments after attempted suicide, the assessment of co-morbid psychiatric conditions, personality and intellectual assessment, behavior modification, pain intervention and psychological assessments prior to dialysis. 
MULTIDISCIPLINARY TEAM - For the modelling of services a staff footprint for inpatient care was drafted using the following ratios: one doctor per ten service users, one doctor on consultation/liaison service, one professional nurse per ten users and one enrolled nurse per five users. The clinical team during the study period however consisted of only one to two professional nurses and one to two enrolled nurses on average per shift per 30 inpatient service users. The team was completed by two consultant psychiatrists, four medical officers/registrars, a clinical psychologist, psychology intern, occupational therapist and a part time social worker. The unfavorable staff ratios for nursing staff continued during the study period and often escalated their work burden to unacceptable levels.

\section{Morbidity and treatment outcome}

IN PATIENT CARE:

1. Demographic profile - The age distribution of the 438 service users admitted during the study period was: 10-19 years $(n=27) 6.2 \%$; $20-29$ years $(n=123) 28.1 \%$; $30-39$ years $(n=119) 27.2 \%$; $40-49$ years $(n=79) 18 \% ; 50-59$ years $(n=50)$ 12.6\%; 60-69 years $(n=20) 4.6 \% ; 70-79$ years $(n=8)$ 1.8\%; not stated ( $\mathrm{n}=7$ ) 1.6\%. The gender distribution was: male $(n=213) 48.6 \%$ and female $(n=225) 51.4 \%$. Race distribution: Black ( $\mathrm{n}=172) 39.3 \%$, Colored $(\mathrm{n}=70) 15.9 \%$, European $(n=145) 33.1 \%$, Indian $(n=37) 8.4 \%$, not stated $(n=14)$.

2. District of origin - Service users from Gauteng Province constituted 399 (91.1\%) of the total; 11 service users were from other provinces, 7 confirmed to be from other African countries and 11 users' district of origin was not stated.

3. Referring unit - The majority of referrals were from the casualty unit ( $\mathrm{n}=317$ ) $72.4 \%$ while the others were from the Department of Medicine ( $n=43$ ) 9.8\%; other hospital departments e.g. Surgery ( $n=34$ ) 7.8\%; community psychiatric clinics $(\mathrm{n}=19)$; Helen Joseph Hospital outpatients $(n=12)$ and the two psychiatric hospitals on the circuit ( $\mathrm{n}=8)$.

4. Reason for admission - Abnormal or inappropriate behaviour - 15\%; violence and aggression 15\%; psychotic symptoms - 17\%; "relapse" - 19\%; suicide attempt - 6\%; substance abuse - $2 \%$; confusion $-4 \%$; depressive symptoms - 10\%; suicidal ideation - 6\%; elevated mood $5 \%$; medical - $1 \%$.

5. Non compliance with treatment - Of the total number, non compliance was confirmed in 206 (47\%) service users leading to readmission, but in 123 cases compliance was not mentioned.

6. Substance abuse - In about $40 \%(n=176)$ of users, active substance abuse was confirmed to play a role in the admission to the unit, while for 88 users substance abuse was not stated.

7. Investigations - Of the routine investigations, HIV testing was highlighted. During this one year period, only 77 (17.6\% of total) service users' HIV status was tested, of which 34 (7.7\% of total) tested positive and 43 negative.

8. Inpatient treatment - Trends in the use of medication for acute sedation included zuclopenthixol acetate, clonazepam and lorazepam. Antidepressants mostly used included citalopram, fluoxetine and mianserin. Antipsychotics routinely used were haloperidol, risperidone, sulpiride and flupenthixol deconoate, while mood stabilizers used were mostly lithium and sodium valproate.

9. Multi-axial diagnoses according to DSM IV - Axis I diagnoses made were bipolar mood disorder $(\mathrm{n}=74)$ 16.9\%; major depressive disorder $(n=50) 11.4 \%$; psychosis due to general medical condition ( $n=35) 8 \%$; psychosis due to substance abuse $(n=38) 8.7 \%$; schizophrenia ( $n=105$ ) 23.9\%; schizo-affective disorder $(n=17) 3.9 \%$ and substance abuse $(n=22)$ 5\%. Other Axis I diagnoses made included: adjustment disorder $(n=10)$; brief reactive psychosis $(n=5)$; delirium $(n=4)$; dementia ( $\mathrm{n}=19)$; dysthymia ( $\mathrm{n}=9$ ); mood disorder due to substance abuse ( $n=12)$; mood disorder due to general medical condition ( $\mathrm{n}=12)$; and V-Code $(\mathrm{n}=7)$ [Figure 1]. Most common Axis II diagnoses made were: mental retardation $(n=19)$, Cluster B personality traits or disorder $(n=50) 24 \%$ and Cluster $\mathrm{C}$ traits or disorder $(\mathrm{n}=12)$. In 135 cases an Axis II diagnosis was deferred.

10. Follow-up arrangements - After discharge from Helen Joseph Hospital, service users were referred for follow-up or transferred to: community psychiatric clinics $(n=112)$ 25.6\%; Sterkfontein Hospital for involuntary care $(n=58)$ 13.2\%; Tara Hospital for extended inpatient care $(n=105)$ 23.7\%; Life Esidimeni for long term care $(n=15) 3.4 \%$ and Helen Joseph Hospital outpatients ( $n=108$ ) 24.7\%.

Fourteen users refused inpatient treatment after initial admission during this time and were discharged without follow-up arrangements, and eight users absconded from the unit. Follow-up arrangements were not stated for 18 service users.

11. Clinician responsible - The total average number of inpatients per month per doctor was nine, well aligned with the initial norm of 1:10 set in the modeling phase.

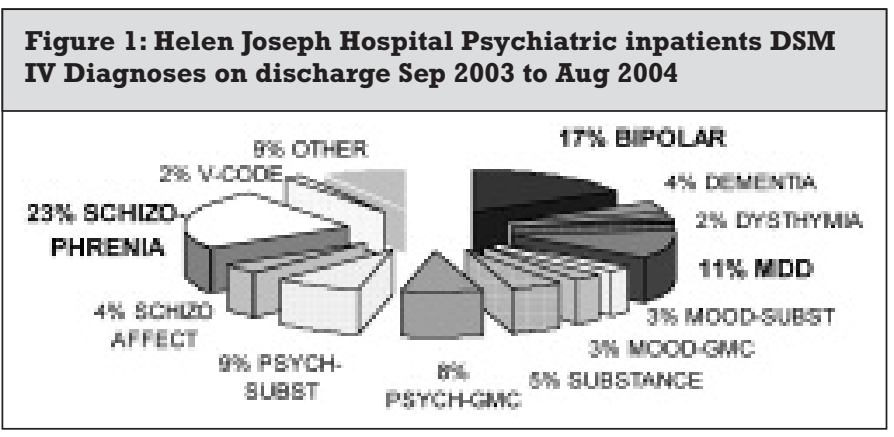

\section{CONSULTATION/LIAISON:}

Data on interdepartmental consultations only represented those undertaken during office hours. Provisional data from routine hospital statistics on after hour consultations from the casualty unit over the study period suggested that at least as many users, about another 40 per month, were referred after hours.

1. Demographic profile - The age distribution of service users consulted during office hours was: 10-19 years $(n=6)$ $7.1 \% ; 20-29$ years $(n=22) 27.4 \% ; 30-39$ years $(n=20)$ $24.8 \%$; 40-49 years $(n=15) 19.1 \%$; $50-59$ years $(n=8) 9.9 \%$; 60-69 years $(n=4) 5.3 \%$; $70-79$ years $(n=2) 3 \%)$; not stated $(n=3) 3.4 \%$. The gender distribution was male $(n=40) 50 \%$ and female $(n=40) 50 \%$. Race distribution: Black ( $n=32)$ 
40\%, Coloured $(n=14) 17.6 \%$, European $(n=25) 31.3 \%$, Indian $(n=4) 5.4 \%$ and not stated $(n=4)$.

2. Referring unit - Referral for consultation was from the casualty unit ( $n=39$ ) 49\%; Department of Medicine ( $n=30)$ $38 \%$; general surgery $(n=5) 6 \%$; other surgical disciplines $(n=3) 4 \%$; other departments $(n=3) 4 \%$.

3. Recommendation after assessment - After consultation service users were referred for follow-up or transferred to: Ward 2 for admission (n=17) 7.1\%; Helen Joseph Hospital psychiatric outpatients ( $\mathrm{n}=30$ ) 37.5\%; follow-up consultation in referring ward ( $\mathrm{n}=13$ ) 15.9\%; Community Psychiatry clinics ( $\mathrm{n}=9$ ) 11.4\%; no follow-up (n=2) 2.5\%; other $(\mathrm{n}=9) 11.4 \%$.

4. Clinician responsible - Twelve doctors rotated through the psychiatric unit during the study period. With one doctor at a time allocated to consultation/liaison on a 2-weekly schedule, the total number of interdepartmental consultations undertaken during office hours therefore was on average 66.6 per doctor.

\section{Discussion}

Focusing, in this portion of the study, only on the service delivery aspect of the clinical audit undertaken at Helen Joseph Hospital, lessons learned about the clinical profile of users and the service profile of the care program included: (i) The majority of service users were either black or European and between 20 and 40 years of age, with an equal gender distribution. (ii) Cross border flow of patients was often not noted and only estimates could be made. Users from other areas often did not state their area of origin especially where foreigners had to be sent back to their countries of origin, which in this study included Ethiopia, Eritrea, Zimbabwe, Angola, Sudan, Somalia, Mozambique and Lesotho. (iii) Referrals for admission were mostly from the hospital's casualty department, followed by the Department of Medicine. (iv) Reasons for admission were most often due to abnormal or inappropriate behavior, violence and aggression, or relapse of symptoms exacerbated by poor compliance and substance abuse. (v) Due to confidentiality issues and inability to grant informed consent, less than one fifth of service users were routinely screened for HIV/AIDS. (vi) Most common DSM IV diagnoses made included schizophrenia, bipolar mood disorder and major depressive disorder. On Axis II, a final assessment of personality structure was often deferred, but diagnoses of Cluster B traits or disorders were made in about a quarter of admissions. (vii) For about 50\% of service users, follow-up arrangements after discharge were mostly made with the ambulatory psychiatric community clinics in the region and with Helen Joseph Hospital's own psychiatric outpatient clinic. This clinical burden resulted generally in an unacceptably high work load expected of nursing staff in particular, following persistent unfavorable nursing staff ratios per service users during the study period.

General lessons learned from the literature about the clinical audit process and problems encountered were gleaned from a number of sources and included Berk et al who reported on the evolution of clinical audit as a tool for quality improvement. ${ }^{1}$ They stated that while the nature of the auditing process is evolutionary, recommendations following on clinical audits were more likely to be implemented if they relied on activity, planning and action across a selection of services. Lyons et al emphasized the importance of the reliability of clinical data for outcome management strategy techniques to be credible. ${ }^{2}$ Balogh and Bond commented on the complexities involved in actually implementing recommendations from clinical audit projects. ${ }^{3}$ It may take up to three years to reach a point at which action is deemed to have been taken. Goldberg gave an overview of the significant business and financial challenges to psychiatric programs in five areas: departmental, hospital, payments system, general finance and policy. ${ }^{4}$ For academic programs, incentive systems were suggested not only addressing clinical productivity, but academic and teaching output as well; general hospital programs were to develop increased sophistication in differential cost accounting; useful reports by leaders in psychiatry needed to be defined and developed to track progress and identify problems; health leaders should be responsible for a strategy to develop the architecture and infrastructure for an appropriate information system. Glover's paper described the pilot work for a mental health database intended to be implemented throughout the English National Health Service and confirmed that monitoring and researching clinical care calls for a comprehensive clinical databases. ${ }^{5}$ Collier and Pyke outlined a model for addressing clinical effectiveness, illustrating how it was implemented through teamwork in a multidisciplinary in-patient environment. ${ }^{6}$ Tobin and $\mathrm{Chen}^{7}$ described the journey of implementing quality improvement activities in the south eastern Sydney area and Wing et al's report ${ }^{8}$ focused on standards of clinical care being achieved for people with schizophrenia in the United Kingdom.

To reconcile these general lessons from the literature within the objectives and results of this study may remain a challenge if clinical audit studies such as this one continue to be only individual and isolated exercises detached from the context of a particular facility's referral and managerial system. To use clinical audit as a tool for quality improvement in our local setting, to have access to reliable clinical data through comprehensive databases across the facilities on the circuit, to successfully implement recommendations from clinical audit projects and to monitor mental health service rendering according to established and acceptable standards, much needs to be done in our sub-region and province to set up regular clinical audit structures, mechanisms and processes. In particular, to monitor service rendering and treatment outcome following the implementation of the current mental health care legislation, effective measuring procedures according to specific norms and standards should routinely be in place. Reports in the literature have highlighted the complexities in actually implementing recommendations from audit reports even if they are made and while the monitoring of achieving objectives happens over time, these procedures and processes cannot be a once off undertaking in the isolation of one or two individual facilities.

Recommendations that can be made in this regard, include: (i) Existing draft policy proposals should urgently be finalized and implemented as policy in view of the new mental health legislation necessitating a sophisticated system of reporting and tracking of service users; (ii) Clinical audit processes and databases for mental health care facilities on the academic and service delivery circuit of the University of 
the Witwatersrand should be integrated and recommendations regularly assessed with relevant feedback to clinicians and managers; and (iii) Although this study was a trial process in one hospital department to implement proposed provincial policy, other sections and departments of Helen Joseph Hospital should also follow in order to report appropriately to the hospital's to be established clinical audit committee.

\section{Conclusion}

Although the current content may provide some baseline for clinical audit processes in comparable mental health care units of other general hospitals in their regional referral context, morbidity and treatment outcome at Helen Joseph Hospital will only be contextualized with the completion of regular related clinical audit studies at the other facilities in its academic and service delivery network as well.

\section{Acknowledgements}

Acknowledgement is given to the medical superintendent of Helen Joseph Hospital and the Faculty of Health Sciences of the University of the Witwatersrand through its Ethical

Committee for granting permission to undertake and report on a preliminary clinical audit of mental health care at the Helen Joseph Hospital.

\section{References}

1. Berk M, Callaly T, Hyland M. The evolution of clinical audit as a tool for quality improvement. J Eval Clin Pract. 2003; 9(2): 251-257.

2. Lyons JS, Rawal P, Yeh I, Leon SC, Tracey P. Use of measurement audit in outcomes management. J Behav Health Serv Res. 2002; 29(11): 75-80.

3. Balogh $R$, Bond $S$. Completing the audit cycle: the outcomes of audits in mental health services. Int J Qual Health Care. 2001; 13(2):135-142.

4. Goldberg RJ. Financial management challenges for general hospital psychiatry 2001. Gen Hosp Psychiatry. 2001; 23(2):62-72.

5. Glover GR. A comprehensive clinical database for mental health in England. Soc Psychiatry Psychiatr Epidemiol. 2000; 35(11): 523-529.

6. Collier EH, Pyke S. Clinical effectiveness on an acute in-patient psychiatric unit. Clin Perform Qual Health Care. 2000; 8 (3): 158-163.

7. Tobin $M$, Chen L. Initiation of quality improvement activities in mental health services. J Qual Clin Pract 1999; 19 (2):11 1-116.

8. Wing JK, Rix S, Cutis RH, Beadsmoore A, Lelliot P. Protocol for assessing services for people with severe mental illness. Br J Psychiatry 1998; 172:121-129.

\section{EDEN MEDICAL RECRUITMENT LTD. CONSULTANTS}

\section{HEAD OF PSYCHIATRY}

\section{SHROPSHIRE, UNITED KINGDOM}

\section{C $\{115 \mathrm{k}$ annual package + head of department allowance + travel allowance}

We are seeking an experienced Psychiatrist with full GMC registration, membership of the Royal College of Psychiatrists and approval under Section 12 (2) MHA 1983.

Our Client, is a modern, purpose-built 43-bed independent Secure Hospital for adults with learning disabilities and challenging behaviour who are detained under the Mental Health Act.

The post-holder will ensure the overall effective coordination and development of clinical services within the hospital and act in this capacity in support of the Hospital Director

Please contact Zita Smith

on 00442077364025 or e-mail your C.V. to

enquiries@edenmedicalrecruitment.co.uk

www.edenmedicalrecruitment.co.uk 